\title{
Measuring Halon Level Based on the Model of Residual Ultrasonic Waves in Container's Wall
}

\author{
Tian Yiyang,Qiu Jinshui \\ College of Naval Architecture and Power \\ Naval Univ. of Engineering \\ WuHan, China
}

\author{
Liu Boyun \\ College of Naval Architecture and Power \\ Naval Univ. of Engineering \\ WuHan, China
}

\begin{abstract}
The halon is usually stored in the sealed highpressure steel containers at liquid state, which can not be detected the liquid level by using the traditional contact-type method. In this paper ultrasonic is used to measure the liquid level which achieved the purpose of nondestructive testing, the model of residual ultrasonic waves in the container's wall was used to determine the liquid level. We choose the wavelet threshold denoising in the wavelet transform to remove the noise in the sampling signals, by comparing the envelope area in two different states that the transducer below or on the actual liquid level to judge the results. It has been proved after repeated tests that this method operates simply, and has a more accurate conclusion.
\end{abstract}

Keywords-halon, the residual ultrasonic waves,liquid level,wavelet denoising

\section{INTRODUCTION}

Because of the excellent actions in extinguishing electrical fires and oil fires, halon is widely used in power plants, electrical rooms and many other mechanical electrical areas. Halon is at liquid state that usually stored in sealed high-pressure steel containers [1],which releasing gaseous materials to the fire rooms by the role of driving gas to extinguish the fire. After each release, we must fill according to the amount of the remaining to prepare for use. Therefore, it has great significance to measure the dose of halon in containers.

The containers are large in size and heavy in weight, so it is not convenient to measure the halon dose by weighing, which has a great workload and may demolish the pipe that may cause leaks. The easiest way to implement dose measurement is to measure the liquid level in containers. Due to the characteristics of high precision, short response time, no harm to human body and easy to site use, ultrasonic is suitable for halon cylinder liquid level detection.

\section{MODEL}

Place the ultrasonic transducer perpendicular to the wall outside of a container, the transmitting ultrasonic waves will generate reflection and transmission effects when through the container's wall[2-3],whether or not the liquid level has reached the point on the wall at which the transducer is attached have different transmission rates, so that the residual ultrasonic waves in the container's wall have different attenuation trends. The model of residual ultrasonic waves take advantage of the principle that the amplitude of ultrasonic signals that bouncing back and forth in the container wall will decay with each round trip or reverbertion through the wall. When the level reached the point at which the sensor is attached, the signals will transmit more to the liquid in the container and decay sharply; if in the side of the detection point is gas, the signals transmit into the gas is the small, relatively decay gently.

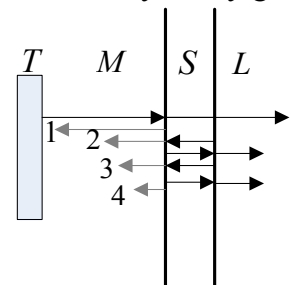

(a) $\mathrm{T}$ - ultrasonic transducer $\mathrm{M}-$ coupling agent, $\mathrm{S}-$ container'wall, $\mathrm{L}$ - medium in container

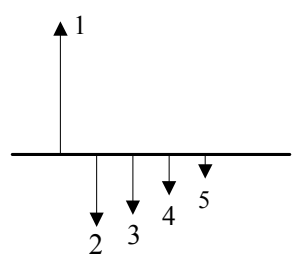

(b) the residual ultrasonic waves in the container's wall

Figure 1. the ultrasonic transducer

As shown in figure1(a),when the ultrasonic transducer transmit pulse signals perpendicular to the container's wall,to the first interface(coupling agent/steel wall) the signals will produce the first echo(figure1(b)), the rest part will transmit into the steel wall, and propagate to the second interface(steel wall-liquid),because of the acoustic impedance suddenly changes, some of the signals pass through and some are reflected back into the wall. We can get from the equation(1), the smaller the impedance mismatch, the less signals are reflected and the more pass through, the greater the mismatch, the more signals reflected back. The residual ultrasonic waves oscillate between the first and second interfaces, transmission will occur when encounter different media interface, the amplitude of the signals gradually attenuate. The signals that transmit through the steel wall-coupling agent interface, will be received by the ultrasonic sensor. 
Therefore, series of reflected pulses are composed of a large initial amplitude of reflected pulse P1 and followed by pulses of certain attenuation coefficient. The amplitude of the signals is decided by the acoustic impedance of coupling agent, steel, liquid/air. $Z_{0} 、 Z_{1}$ and $Z_{2}$ indicate the acoustic impedance of coupling agent, steel, liquid/air respectively. The first amplitude[4] of the echo is

$$
P_{1}=\frac{Z_{1}-Z_{0}}{Z_{1}+Z_{0}}
$$

The ratio of second echo with the first is

$K_{R}=\frac{P_{2}}{P_{1}}=\frac{4 Z_{0} Z_{1}\left(Z_{2}-Z_{1}\right)}{\left(Z_{1}-Z_{0}\right)\left(Z_{1}+Z_{2}\right)\left(Z_{1}+Z_{0}\right)}$

$P_{1}$ and $P_{2}$ indici

echo pulse respectively. The attenuation rate[5] of the following echo pulses is

$$
K_{A}=\frac{P_{3}}{P_{2}}=\frac{P_{N+1}}{P_{N}}=\frac{\left(Z_{2}-Z_{1}\right)\left(Z_{0}-Z_{1}\right)}{\left(Z_{2}+Z_{1}\right)\left(Z_{0}+Z_{1}\right)}, \quad N \geq 2
$$

From the above equation(3) can be drawn: It is obviously that when the acoustic impedance of coupling agent or the liquid is close to the acoustic impedance of container's wall. A smaller value of $K_{A}$ indicating that the greater attenuation of pulse amplitude. After the ultrasonic pulse oscillates several round and forth, the amplitude of the ultrasonic signal which transducer receives become very small. Based on this point, processing the first few (up to 6-7)echo signals which have better signal to noise ratio is most reasonable. Calculate the envelope area of the residual ultrasonic waves, if the transducer on the wall is higher than the actual liquid level, the envelope area is larger. Usually, the ratio of the two states area envelope area is $2: 1$ or more.

\section{DATA COLLECTION}

Considering the steel type of halon containers and debugging of repeated tests, we choose an all digital multichannel ultrasonic board CUT-2001, a ultrasonic transducer with transmitting frequency of $1.25 \mathrm{MHz}$,glycerol and $\mathrm{PC}$ to complete the data analysis. The block design diagram is shown in Figure 2.

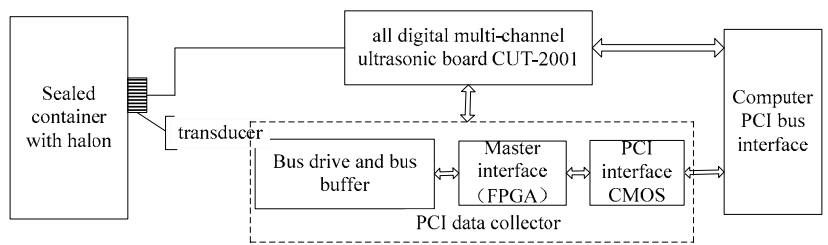

Figure 2. block design diagram of the collecting system

The test platform is shown in Figure 3 below.

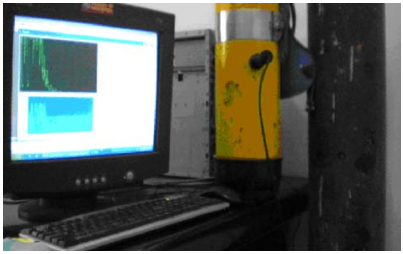

Figure 3. The platform for testing

The acquired signal is shown in figure 4 below.Fig.4(a) shows a echo wave of the steel-vapor interface,Fig.4(b) shows wave of the steel-liquid interface.

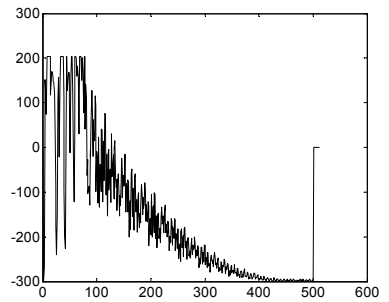

(a) a echo wave of the steel-vapor interface

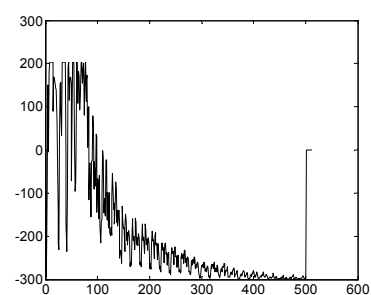

(b) a echo wave of the steel-liquid interface

Figure 4. The acquired signal is shown

\section{Signal Processing AND JUdGEMENT}

The circuits, materials of container and many other reasons introduces lot's of noise in the ultrasonic test, which will influence the subsequent judgments and bring on unpredictable interference to the result, so we have to give a deeper analysis to the collected ultrasonic echo signals so as to improve the detection accuracy and reliability. In this paper, the wavelet analysis is choosed for processing the signals, which can decompose the signals into different frequency bands. We choose the frequency components needed for further analysis. Threshold denoising method based on wavelet transform is composed of four parts [6]:the selection of wavelet function, decomposition of the signal acquisition, selection of threshold and signal reconstruction.

Daubechies wavelets are the wavelets typically smooth with compact support orthogonal [7],we choose db5 wavelet for decompose the sampling data.

Sampling signal $s=a 3+d 3+d 2+d 1$, in which, $a 3$ is the similar signal to the third level decomposition, $d 1, d 2$ and $d 3$ are the detail signals on the first, second, third level decomposition, respectively. 

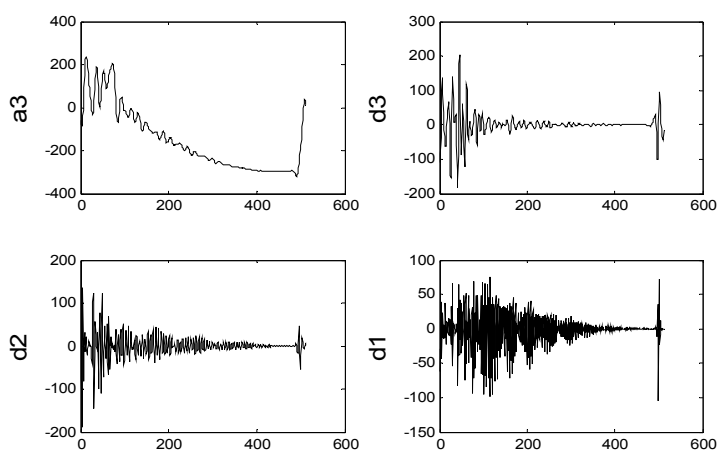

Figure 5. signal decomposing

Wavelet threshold denoising method has two kinds: first, given the threshold denoising method, also known as softthreshold method; second, mandatory noise reduction method, also known as a hard threshold method, that is to set all of the high frequency coefficients 0.According to actual situation, in this paper, we just need the decay process of the residual ultrasonic waves to be clearly reflected, so choose hard-threshold denoising method, its flow chart is shown in Figure 6.

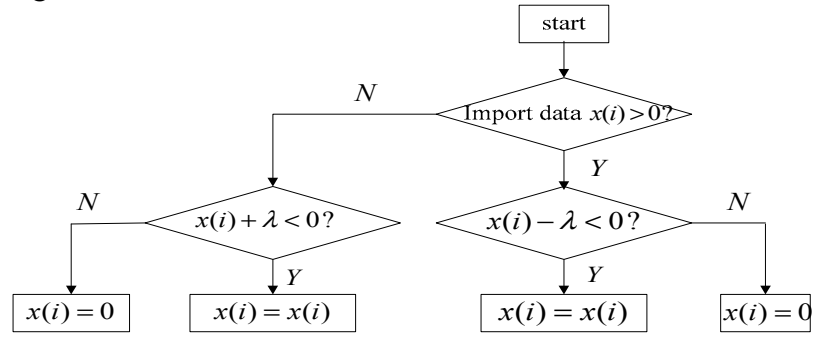

Figure 6. chart of filtering program

The residual ultrasonic waves of the steel-vapor interface after denoising is shown in figure 7(a).The acquired signals are composed of transmission pulse signals and decay part, we set $t_{1}$ in the figure as Ping Value, based on data access[8],Ping value $=$ time length of transmit pulses, by the end of the transmitting pulse, the decay process in the figure is the residual ultrasonic waves in the container's wall. We just about to distinguish the transducer whether or not reaches the liquid level by the attenuation characteristics. Figure 7(a) and 7(b) show the attenuation process of the residual ultrasonic waves that the transducer

above or below liquid level, respectively. The shadow part of figure represents the process of signal attenuation and the envelope area of the attenuation signal.

We set $S_{\operatorname{Re} f}$ as the threshold of the envelope area, if the detected envelope area is larger than $S_{\text {Ref }}$, that indicates the point at which level the transducer attached in the container is vapor, contrarily, if the area is smaller than $S_{\operatorname{Re} f}$, it is liquid in the container. Set the envelope area in figure 7(a) as $S_{E}$, in figure 7(b) as $S_{F}$, the threshold range can be set by the following formula

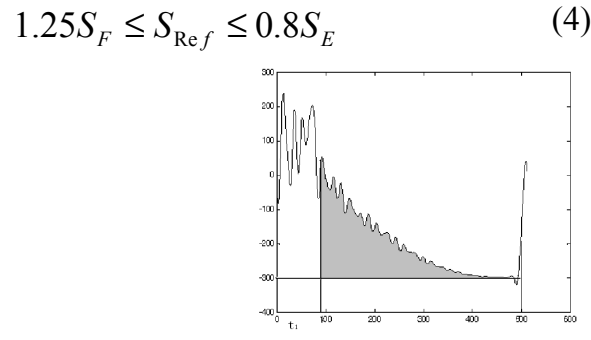

(a) the envelope area of the steel-vapor interface

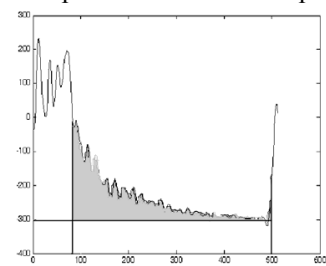

(b) the envelope area of the steel-liquid interface

Figure 7. Set the envolope area

\section{CONCLUSION}

In the traditional ultrasonic level measurement, the transducer is difficult to install, also has difficulties of positioning. This method of ultrasonic liquid level measurement in this paper is easy to operate, the proposed method that based on the envelope area of the residual ultrasonic waves is more accurate, and achieved good results. We can develop interface through Visual Basic, Visual C ++ ,call Matlab for signal denoising and judgments, so as to simplify the operation.

\section{REFERENCES}

[1] Zheng Ruiwen, Liu Haichen. Fire Fighting Safty [M]. Beijing : Chemical Industry Press, 2005

[2] Ma Zhimin, Zhao Xiaohong. Analysis of attenuation characteristics of residual ultrasonic waves in container wall and its application [J]. Acoustics Technology : 2003, 22(4) : 233-235

[3] Ma Zhimin. Study on the method of measuring fixed-point liquid level based on ultrasonic penetrative attenuation in the wall of containers[J]. Research and Exploitation:2003,4:45-46,49

[4] Chen Lei, Dong Xinmin, Han jie, Ye Ping. Development of ultrasonic instrument for sealed container's liquid level measurement [J]. ULTRAGARSAS : 2005, 3(56) : 30-35

[5] Sidney G.Allison . Ultrasonic Measurement of Aircraft Strut Hydraudic Fluid level $[\mathrm{J}]$. NASA Langley Research Center : 02WAC-19

[6] Li Qiang. Data Sampling and Signal Processing of a Distributed Polarization Coupling Analyzer [D]. Tianjin University, 2005

[7] Yi Xiong. Mechanical Faults Feature Extraction and Diagnostic Techniques Research Based on Wavelet Analysis [D]. Zhejiang University of Technology, 2009

[8] Zhang Chao. The Enverlop Method of Echoed Tail Wave Used in the Classification of Sediment in the Dissertation [D]. Central South University, 2007 